\title{
Title: ChAdOx1 NiV vaccination protects against lethal Nipah Bangladesh virus infection in African green monkeys
}

Authors: Neeltje van Doremalen ${ }^{1}$, Victoria A. Avanzato ${ }^{1}$, Friederike Feldmann ${ }^{2}$, Jonathan E.

Schulz $^{1}$, Elaine Haddock ${ }^{1}$, Atsushi Okumura ${ }^{1}$, Jamie Lovaglio ${ }^{2}$, Patrick W. Hanley ${ }^{2}$, Kathleen

Cordova $^{2}$, Greg Saturday ${ }^{2}$, Teresa Lambe ${ }^{3}$, Sarah C. Gilbert ${ }^{3}$, Vincent J. Munster ${ }^{1 *}$

\section{Affiliations:}

${ }^{1}$ Laboratory of Virology, National Institute of Allergy and Infectious Diseases, National

Institutes of Health, Hamilton, MT, USA

${ }^{2}$ Rocky Mountain Veterinary Branch, National Institute of Allergy and Infectious Diseases,

National Institutes of Health, Hamilton, MT, USA

${ }^{3}$ The Jenner Institute, Nuffield Department of Medicine, University of Oxford, Oxford, UK

*To whom correspondence should be addressed: vincent.munster@nih.gov

One Sentence Summary: A single vaccination with ChAdOx1 NiV protects African green monkeys against lethal disease induced by Nipah virus inoculation. 
1 Abstract: Nipah virus (NiV) is a highly pathogenic and re-emerging virus which causes sporadic

2 but severe infections in humans. Currently, no vaccines against NiV have been approved. We

3 previously showed that $\mathrm{ChAdOx} 1 \mathrm{NiV}$ provides full protection against a lethal challenge with

4 NiV Bangladesh (NiV-B) in hamsters. Here, we investigated the efficacy of ChAdOx1 NiV in

5 the lethal African green monkeys (AGMs) NiV challenge model. AGMs were vaccinated either 4

6 weeks before challenge (prime vaccination), or 8 and 4 weeks before challenge with ChAdOx1

$7 \quad \mathrm{NiV}$ (prime-boost vaccination). A robust humoral and cellular response was detected starting 14

8 days post initial vaccination. Upon challenge, control animals displayed a variety of signs and

9 had to be euthanized between 5- and 7-days post inoculation. In contrast, vaccinated animals

10 showed no signs of disease, and we were unable to detect infectious virus in all but one swab

11 and all tissues. Importantly, no to limited antibodies against fusion protein or nucleoprotein IgG

12 could be detected 42 days post challenge, suggesting that vaccination induced a very robust

13 protective immune response preventing extensive virus replication. 


\section{Introduction}

Nipah virus (NiV) is a highly pathogenic re-emerging member of the Paramyxovirus family, genus Henipavirus. NiV causes sporadic infections in humans, resulting in severe neurological and respiratory disease, often with a fatal outcome. NiV was first detected in 1998, when the strain NiV-Malaysia (NiV-M) caused an outbreak of severe encephalitis in pig farmers from Malaysia and Singapore, with a case-fatality rate of $38 \%{ }^{1}$. Since 2001 , outbreaks with a closely related strain, NiV-Bangladesh (NiV-B), have occurred almost yearly in Bangladesh ${ }^{2}$, resulting in 319 accumulated cases and 225 associated deaths (case-fatality rate $71 \%)^{3}$. Most recently, outbreaks have also been reported in India ${ }^{4}$.

The natural reservoir of NiV is the Pteropus spp. fruit bat ${ }^{5-7}$. Outbreaks in Bangladesh and India have been associated with the consumption of date palm sap, which may have been contaminated with bat urine ${ }^{8-10}$. In contrast, in Malaysia and Singapore, pigs were the intermediate host, likely infected via the consumption of mango fruits, which were contaminated with NiV after partial consumption by bats ${ }^{11}$. Importantly, human-to-human transmission of NiV has also been reported $^{12,13}$.

Although the total number of cases caused by NiV are limited, the virus causes severe disease and transmits between humans ${ }^{12,13}$, and can infect a wide range of animals ${ }^{14}$, and thus NiV is categorized by the WHO as a pathogen with epidemic potential which poses a great public health risk and requires research aimed at the development of countermeasures.

Several vaccine candidates have been evaluated in animal models ${ }^{15}$. The most extensively studied vaccine to date is based on the glycoprotein of Hendra virus $(\mathrm{HeV})$, another member of the genus Henipavirus ${ }^{16}$. $\mathrm{HeV}-\mathrm{sG}$, a soluble form of the $\mathrm{HeV}$ receptor binding glycoprotein, was marketed by Zoetis, Inc., in Australia as an equine vaccine against $\mathrm{HeV}$ under the name Equivac ${ }^{\circledR} \mathrm{HeV}$. It is the first commercialized vaccine against a BSL-4 agent ${ }^{17}$. Recently, it was shown that $\mathrm{HeV}-\mathrm{sG}$ vaccination can protect African green monkeys (AGMs) against lethal NiV disease as early as 7 days post immunization ${ }^{17}$. Enrollment has started for a Phase I randomized placebo-controlled clinical trial on NiV-vaccine candidate $\mathrm{HeV}$-sG-V, which is based on HeV-sG, with results expected in October 2021 (ClinicalTrials.gov NCT04199169). This clinical trial is the first of its kind for $\mathrm{NiV}$ and is the result of a global partnership between Auro Vaccines LLC and the Coalition of Epidemic Preparedness Innovations (CEPI). 
In the current study, we are testing efficacy of a different NiV vaccine candidate in AGMs. ChAdOx1 is a replication-deficient simian adenoviral vector, which has been developed for a multitude of different pathogens by the University of Oxford. A vaccine based on this vector named ChAdOx1 nCoV-19 (also known as AZD1222, Vaxzevria, or Covishield) has been developed against severe acute respiratory syndrome coronavirus 2 (SARS-CoV-2), the etiological agent of COVID-19. ChAdOx1 nCoV-19 has been fully approved in Brazil and approved for emergency use in 64 additional countries. The effectiveness of the vaccine is $92 \%$ and is $79 \%$ against symptomatic infection ${ }^{18,19}$.

We have previously demonstrated that a single dose of ChAdOx1 NiV, which encodes the receptor binding protein $(\mathrm{G})$ of NiV-B, fully protected Syrian hamsters against a lethal dose of $\mathrm{NiV}-\mathrm{B}$ or $\mathrm{NiV}-\mathrm{M}^{20}$. In the current study, we investigate whether $\mathrm{ChAdOx} 1 \mathrm{NiV}$ is protective in the lethal NiV AGM model ${ }^{21}$. We show that a single dose of ChAdOx1 NiV results in a robust innate and adaptive immune response, which is fully protective against lethal disease in AGMs. Furthermore, no to limited immune response against nucleoprotein or fusion protein was detected in vaccinated animals post challenge, but was detected in control challenged animals, suggesting that vaccination provides near complete protection against NiV infection.

\section{Results}

ChAdOxl NiV vaccination of African green monkeys elicits a potent adaptive immune response Four animals per group were vaccinated via the intramuscular (I.M.) route with ChAdOx1 NiV using a prime-boost regimen at 56 and 28 days before challenge, or a prime-only regimen at 28 days before challenge. As a control, four animals were vaccinated via the I.M. route with ChAdOx1 GFP at 56 and 28 days before challenge. Binding antibody titers against NiV G protein were determined at day of vaccination, 14 days post vaccination, and day of challenge. NiV G-specific IgG antibodies could be detected as early as 14 days post vaccination and were significantly increased upon boost vaccination (Two-tailed Mann-Whitney test, $\mathrm{p}=0.0286$ ). All vaccinated animals had detectable NiV G-specific antibodies on the day of challenge (Figure 1A). No NiV G-specific IgG antibodies were detected in control animals. Likewise, virus neutralizing antibodies were detected in all vaccinated animals at day of challenge and were significantly increased upon boost vaccination (Figure 1B, Mann-Whitney test, $\mathrm{p}=0.0286$ ). NiV G-specific T cell responses were investigated using a peptide library divided into six peptide 
A

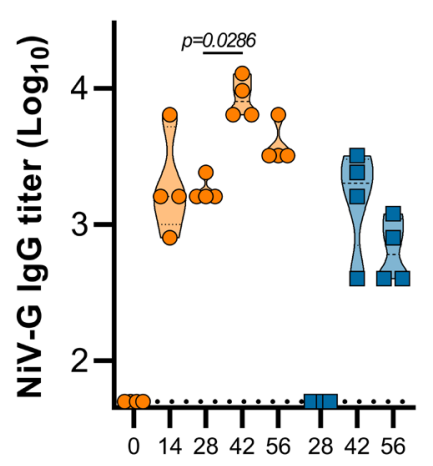

Days post vaccination
B

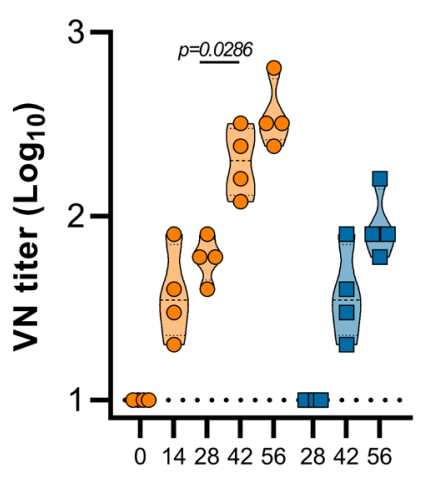

Days post vaccination
C

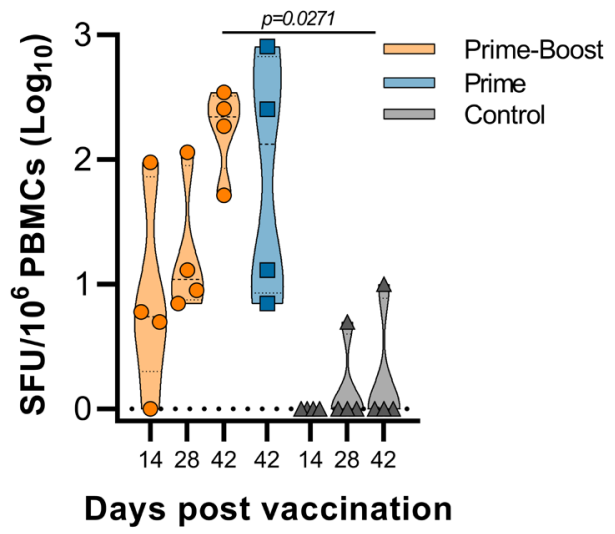

Figure 1. Vaccination with ChAdOx1 NiV in AGMs induces humoral and cellular immune responses. (A) Truncated violin plot shows evidence of $\mathrm{NiV}-\mathrm{G}$-specific IgG in serum at the indicated days post prime vaccination in animals receiving intramuscular ChAdOx1 NiV via a prime-boost (orange, $n=4$ ) or prime-only regimen (blue, $n=4$ ). (B) Truncated violin plot of neutralizing antibodies in serum are shown. (C) Truncated violin plot of NiV-G protein-specific $\mathrm{T}$ cell responses in PBMCs isolated from vaccinated or controls animals at indicated time points minus 0 days post vaccination (DPV) response. SFU, spot-forming units. Black lines indicate median; dotted lines indicate quartiles. The dotted line shows the limit of detection.

Vaccinated animals do not show signs of disease

Animals were inoculated with $2 \times 10^{5} \mathrm{TCID}_{50}$ of NiV-B, split equally between the intranasal and intratracheal route. Animals were checked daily for clinical signs. Animals vaccinated with ChAdOx1 GFP displayed a variety of signs starting at 3 days post inoculation (DPI), including neurological signs and respiratory signs (Table S1). All four animals in this group reached an endpoint clinical score of 35 or higher between 5 to 7 DPI and were euthanized. In contrast, no signs of disease were observed in animals vaccinated with ChAdOx1 NiV (Figure 2A-B, Table S1). Exams were performed on 0, 3, 5, 7, 10, 14, 21, 28, 35, and 42 DPI. Radiographs were scored as previously described ${ }^{22}$. Whereas no to limited changes from baseline were observed in ChAdOx1 NiV vaccinated animals (score between 0-2), radiograph scores of control-vaccinated animals started increasing at 3 DPI (score between 0-3) and continued to increase until day of 
101

102

103

104

105

106

107

108

109

110

necropsy (score between 7-14, Figure 2C). Throat and nose swabs were collected on all exam days and the presence of infectious virus was investigated. Infectious virus could be detected in both nose and throat swabs of 3 out of 4 control-vaccinated animals. In contrast, all swabs obtained from animals vaccinated with $\mathrm{ChAdOx} 1 \mathrm{NiV}$ were negative, except for one throat swab obtained at 3 DPI from one animal (animal 1) in the prime-boost group (Figure 2D-E). The presence of binding antibodies against fusion glycoprotein $(\mathrm{F})$ and nucleoprotein $(\mathrm{N})$ of $\mathrm{NiV}$ was then investigated in sera obtained from vaccinated animals at 42 DPI. A low titer of binding antibodies against $\mathrm{N}$ was found in sera from animal 1, and antibodies against $\mathrm{F}$ were detected in sera from animal 1, 7, and 8 (Figure $2 \mathrm{~F}$ ).
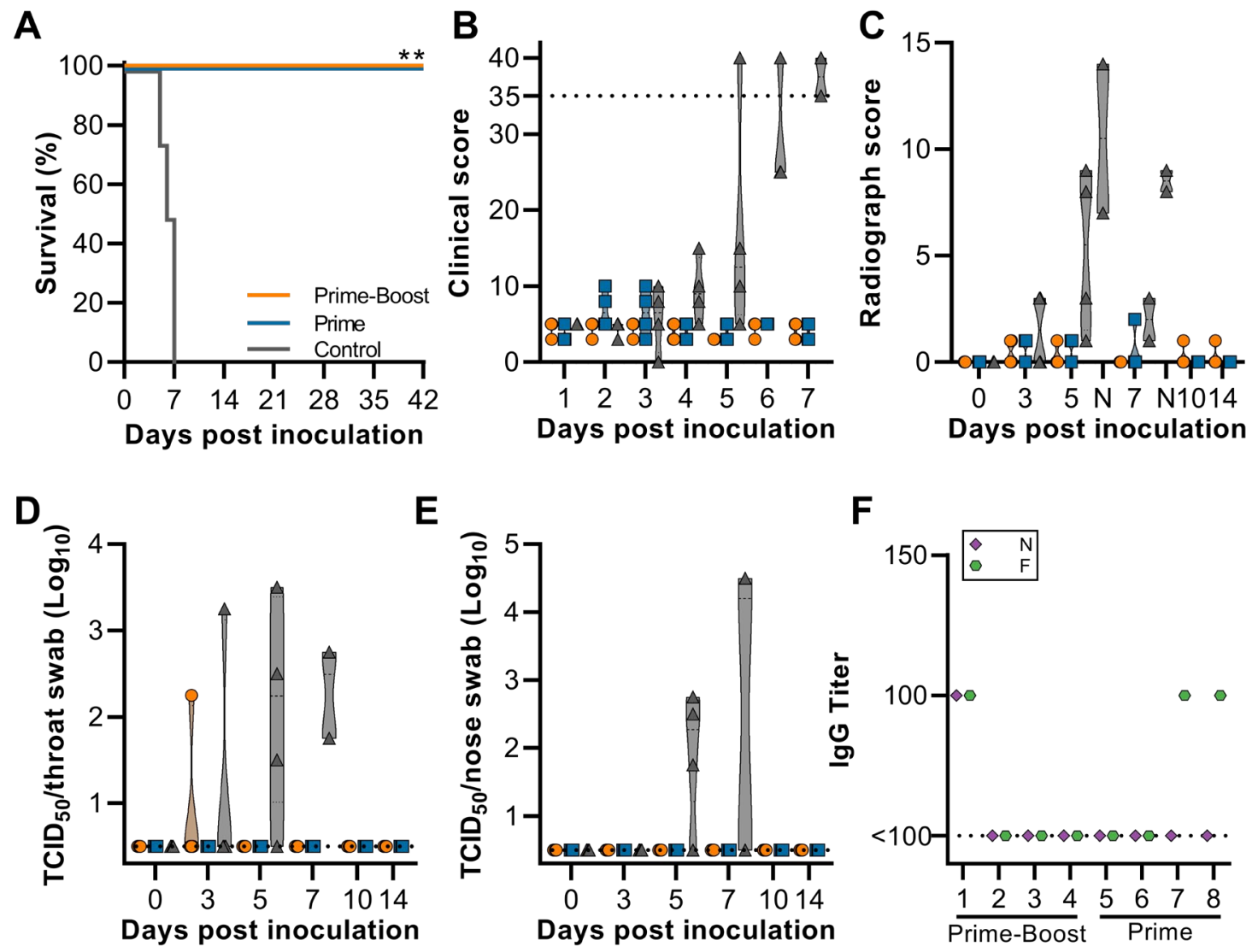

E
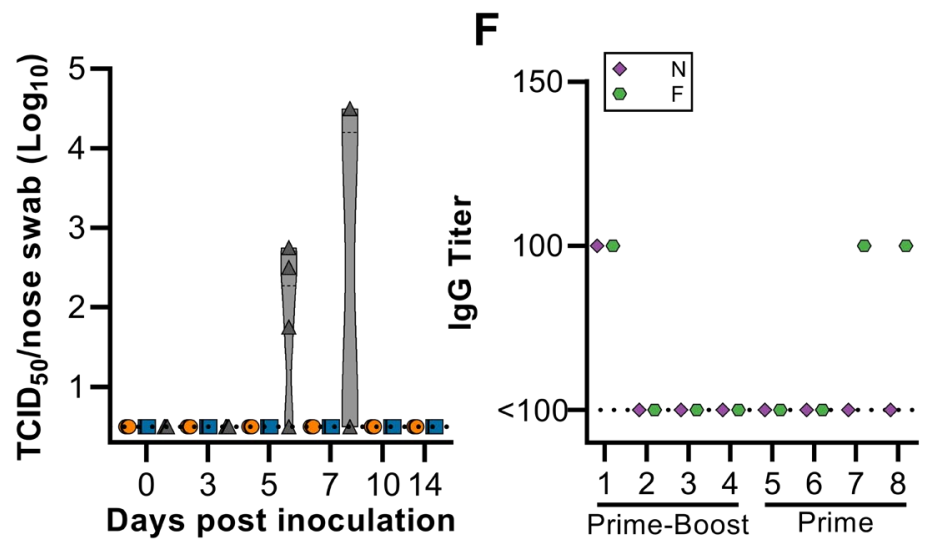

Animal

111
Figure 2. Clinical signs and $\mathrm{NiV}$ detection in vaccinated AGMs upon virus challenge. 28 days post final vaccination, AGMs ( $\mathrm{N}=4$ per group) were inoculation with $\mathrm{NiV}-\mathrm{B}$ and monitored for up to 42 days. (A) Survival of AGMs. (B) Truncated violin plot of daily clinical score. Dotted line indicates score requiring euthanasia. (C) Truncated violin plot of thoracic radiograph scores on exam days and day of necropsy (N, controls only). (D/E) Truncated violin plots of infectious 
$117 \mathrm{NiV}$ in throat swabs (D) and nose swabs (E). At 7 DPI, only 2 control animals were part of the

118 study. Dotted line indicates limit of detection. (F) Truncated violin plot shows lack of evidence

119 of NiV-nucleoprotein (N, purple diamond) or NiV- fusion glycoprotein (F, green octagon) -

120 specific IgG in serum at 42 DPI. Dotted line indicates limit of detection. For all panels, orange

121 indicates prime-boost vaccinated animals, blue indicates prime only vaccinated animals, and

122 grey indicates control animals.

123

No evidence of $\mathrm{NiV}$ detected in tissues of vaccinated animals

125 Animals were euthanized when euthanasia criteria were reached (control group, 5-7 DPI) or at

12642 DPI (end of study, vaccinated animals). Lung:body weight ratio, indicative of edema, was

127 higher for control animals than for vaccinated animals (Figure 3A). At necropsy, the percentage

128 of each lung lobe that showed lesions was scored. Whereas a high percentage of gross lesions

129 was observed in control animals (50 out of 56 lung lobes, median 50\%), this was almost

130 completely absent in vaccinated animals (9 out of 56 lung lobes, median $0 \%$ for both prime-

131 boost and prime-only vaccinated groups) (Figure 3B). Lungs of control animals failed to collapse

132 (3 out of 4), and pleural effusion was observed in all animals. Cervical lymph node enlargement

133 and edematous mediastinal lymph nodes were observed in 2 and 3 out of 4 control animals,

134 respectively. One animal showed a diffuse hemorrhage from the medulla oblongata to the

135 cervical spinal cord, with petechiae on the cerebellum.

136

137

138

139

140

141

142

143

144

145

146
Histologically, no pulmonary pathology consistent with NiV lesions was observed in vaccinated animals (Figure 4A-B), and subsequently the pathology score was low (Figure 3C). Likewise, no NiV RNA staining was detected in lung tissue (Figure 4D-E). In stark contrast, histological lesions were present in lung tissue obtained from all control animals and were characterized as multifocal, random, minimal (1-10\%) to marked (51-75\%) bronchointerstitial pneumonia. The pneumonia was characterized by thickening of alveolar septa by edema fluid and fibrin and small to moderate numbers of macrophages, syncytial cells and neutrophils (Figure 4C). In situ hybridization reveals abundant viral RNA distributed throughout lesions in vascular endothelium and type I\&II pneumocytes in tissue from control animals (Figure 4F). Infectious virus was only detected in tissue obtained from control animals, and not in tissue obtained from vaccinated animals (Figure 3D, S1). 
A

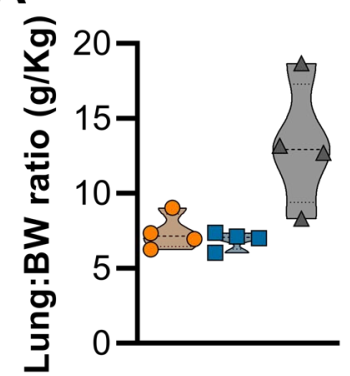

B

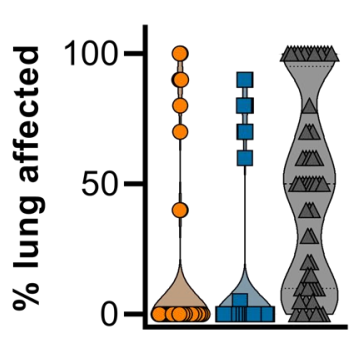

C

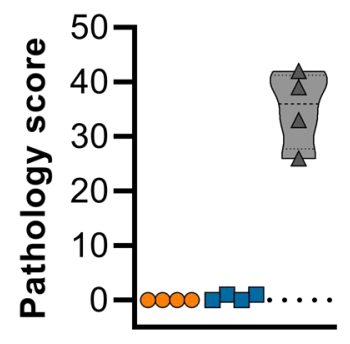

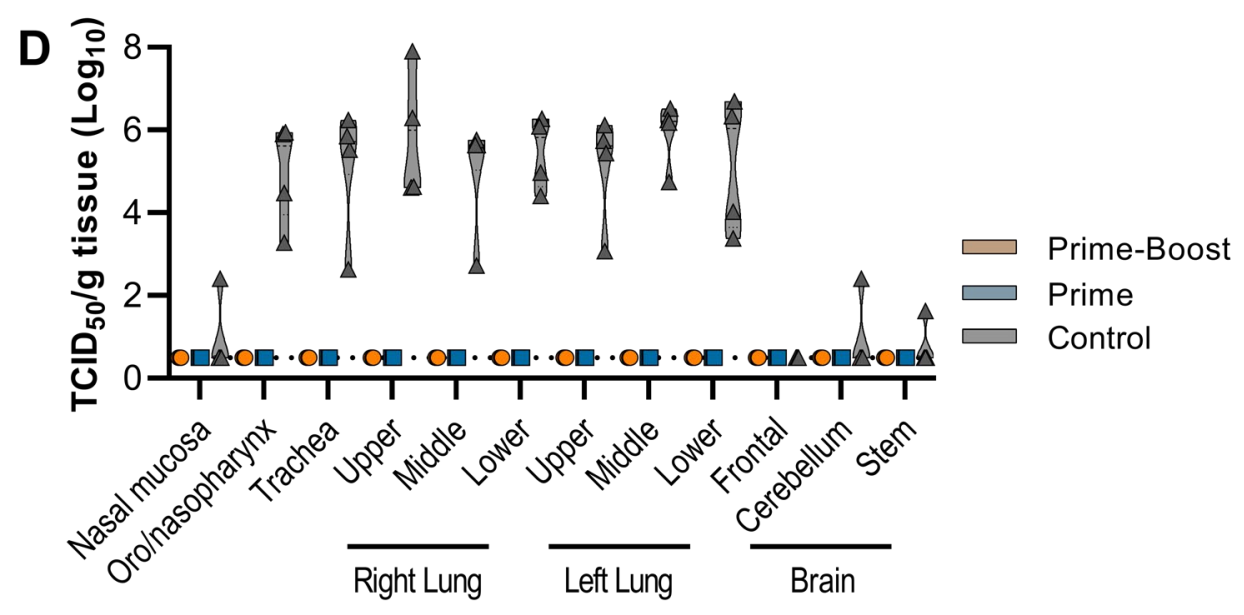

147

Figure 3. No evidence of NiV infection detected in vaccinated animals. AGMs were euthanized when a clinical score of 35 was reached (all control animals, 5-7 DPI) or on 42 DPI (all vaccinated animals). (A) Truncated violin plot of lung:body weight (BW) ratio. (B) Truncated violin plot of gross lung lesions. which were scored for each lung lobe, ventral and dorsal. (C) Truncated violin plot of pathology score. All six lung lobes were scored and cumulative score is shown per animal (maximum score $=90$ ). (D) Truncated violin plot of infectious virus detected in respiratory tract and brain tissue. For all panels, orange indicates prime-boost vaccinated animals, blue indicates prime only vaccinated animals, and grey indicates control animals. No statistical tests were performed since samples were obtained on different days post challenge. 


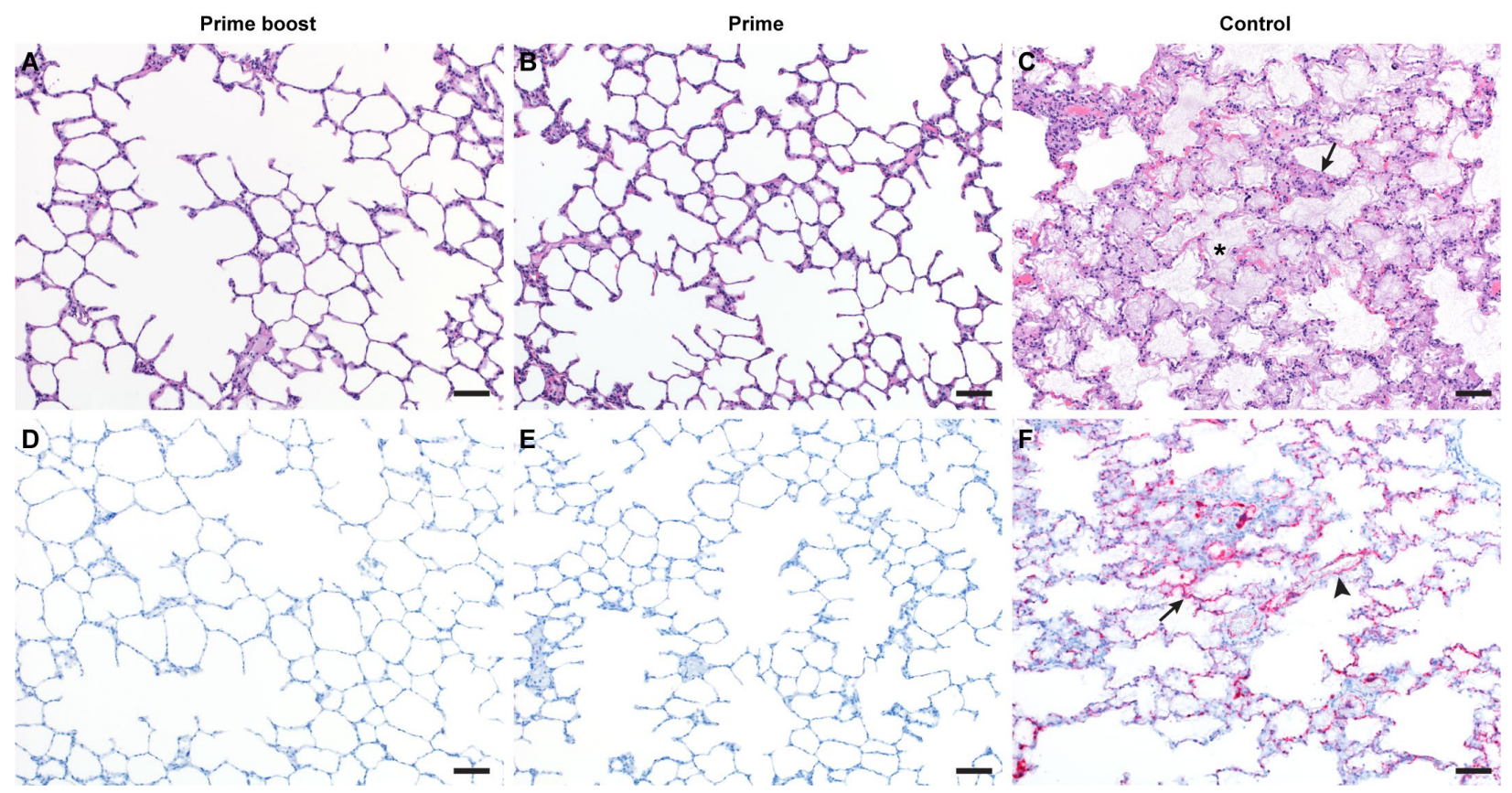

Figure 4. Pulmonary effects of ChAdOx1-NiV vaccine efficacy in the AGM model of NiV infection. (A-C) Lung tissue sections were stained with hematoxylin and eosin. (A-B) no pathology was observed. (C) moderate to marked interstitial pneumonia (arrow) with abundant fibrin and edema (asterisk). (D-F) In situ hybridization for NiV RNA, resulting in a red stain. (DE) no immunoreactivity was observed. (F) Immunoreactive vascular endothelium (arrowhead) and pneumocytes (arrow). 100x bar $=50 \mu \mathrm{m}$.

\section{Discussion}

We evaluated the vaccine candidate $\mathrm{ChAdOx} 1 \mathrm{NiV}$, which we previously showed to be fully protective in Syrian hamsters $^{20}$, in the lethal AGM NiV challenge model. We show here that a single dose of ChAdOx1 NiV was fully protective against a lethal challenge with NiV-B in AGMs. Furthermore, we found very limited evidence of virus replication in vaccinated animals; all but one swab was negative for infectious virus, no virus was found in tissues obtained from vaccinated animals, and no to a very limited immune response was detected against NiV F or $\mathrm{N}$ proteins after challenge of vaccinated animals. These data suggest the vaccine may provide close to complete protective immunity in AGMs.

Bossart et $a l .{ }^{23}$ show a similar lack of NiV-F specific antibodies in vaccinated animals. In that study, AGMs were vaccinated with a HeV-G subunit vaccine 6 and 3 weeks before challenge with a lethal dose of NiV. Serum was obtained throughout the experiment and NiV F-specific IgM could not be detected at any point. Furthermore, whereas vaccination induced high IgG titers against NiV-G and HeV-G, there was no increase in G-specific IgG titers after challenge 
181

182

183

184

185

186

187

188

189

190

191

192

193

194

195

196

197

198

199

200

201

202

203

204

205

206

207

208

209

210

211

with NiV. Together, these data suggest a lack of virus replication in vaccinated animals ${ }^{23}$.

Likewise, in a study performed by Lo et al. ${ }^{24}$, hamsters were vaccinated with replicationdeficient VSV-based NiV G or NiV F. VN titers were measured in serum obtained at 28 days post vaccination and 32 days post challenge. No anamnestic response was detected in vaccinated animals, suggesting that this vaccine may also provide (near) complete proimmunity ${ }^{24}$. In a study by Prescott et al., AGMs were vaccinated with rVSV-EBOV-GP-NiVG, 29 days before challenge with NiV Malaysia. An increase in binding and neutralizing antibodies titer between 0 DPI and 16/17 DPI (termination of study) was found in $2 / 3$ vaccinated animals ${ }^{25}$.

The approval of a NiV vaccine is hindered by the feasibility of an efficacy trial due to the sporadic nature of infections, the large geographical area where the spillover occurs and the low number of annual cases ${ }^{26}$. To address these complications, the U.S. Food and Drug Administration implemented the 'Animal Rule' in $2002^{27}$. This rule can be utilized to establish efficacy based on studies performed in animal models that faithfully recapitulate human disease, such as the AGM and hamster NiV model ${ }^{26}$. Thus far, 18 products have been approved via this route $^{28}$. In both hamster and AGM NiV models, vaccination with ChAdOx1 NiV resulted in induction of high antibody titers coupled with complete protection against lethal NiV disease.

ChAdOx1 NiV is based on the same vector as ChAdOx1 nCoV-19, which has been approved for emergency use in over 60 countries worldwide. Subsequently, 100 million people have been vaccinated with the vector. Safety profiles obtained in ChAdOx $1 \mathrm{nCoV}-19$ clinical studies ${ }^{29}$ combined with efficacy studies in animal models ${ }^{20}$ may provide sufficient information for approval of ChAdOx1 NiV.

Although several NHP studies have successfully investigated the efficacy of NiV vaccines ${ }^{23,25,30-}$ ${ }^{32}$, thus far only one vaccine has advanced into clinical trials. Here, we show that the widely used ChAdOx1 vector can be modified to provide protection against NiV in a lethal NHP model. Previously, similar work investigating the efficacy of a ChAdOx1 MERS vaccine in rhesus macaques $^{33}$ was instrumental in the development of the ChAdOx1 nCoV-19 vaccine ${ }^{34}$. If the next pandemic were to be caused by a member of the genus Henipavirus, the current study could be influential in the development of a rapid vaccine. Future studies, such as Phase I clinical trial studies and further efficacy studies in AGMs, should aim to obtain approval via the FDA Animal Rule. 


\section{Materials and Methods}

\section{Ethics Statement}

214 The Institutional Animal Care and Use Committee (IACUC) at Rocky Mountain Laboratories approved 215 all animal study requests, which were conducted in an Association for Assessment and Accreditation of 216 Laboratory Animal Care (AAALAC)-accredited facility, following the basic principles and guidelines in 217 the Guide for the Care and Use of Laboratory Animals 8th edition, the Animal Welfare Act, United States 218 Department of Agriculture and the United States Public Health Service Policy on Humane Care and Use 219 of Laboratory Animals.

220 Animals were kept in climate-controlled rooms with a fixed light/dark cycle (12-hours/12-hours). African 221 green monkeys were housed in individual primate cages allowing social interactions, fed a commercial 222 monkey chow, treats and fruit with ad libitum water and were monitored at least twice daily.

223 Environmental enrichment consisted of a variety of human interaction, commercial toys, videos, and 224 music. The Institutional Biosafety Committee (IBC) approved work with infectious Nipah virus strains 225 under BSL4 conditions. All sample inactivation was performed according to IBC approved standard 226 operating procedures for removal of specimens from high containment ${ }^{35,36}$.

\section{Study design animal experiments}

22812 African green monkeys $(6 \mathrm{M}, 6 \mathrm{~F})$ between 3-5 years old were sorted by sex, then by weight, and then randomly divided into three groups of four animals. Group 1 was vaccinated with ChAdOx1 NiV at -56 and -28 DPI, group 2 was vaccinated with ChAdOx1 NiV at -28 DPI, group 3 was vaccinated with ChAdOx1 GFP at -56 and -28 DPI. All vaccinations were done intramuscularly with $2.5 \times 10^{10} \mathrm{VP} / \mathrm{animal}$ diluted in sterile PBS. Animals were challenged with Nipah Bangladesh (AY988601) diluted in sterile DMEM at 0 DPI; $4 \mathrm{~mL}$ intratracheally $\left(2.5 \times 10^{4} \mathrm{TCID}_{50} / \mathrm{mL}\right)$ and $1 \mathrm{~mL}$ intranasally $\left(1 \times 10^{5}\right.$ $\mathrm{TCID}_{50} / \mathrm{mL}$ ). Animals were scored daily by the same person who was blinded to study group allocations using a standardized scoring sheet ${ }^{37}$. Scoring was based on the following criteria: general appearance, skin and coat appearance, discharge, respiration, feces and urine appearance, appetite, and activity. Clinical exams were performed on -56, -42, -28, -14, 0, 3, 5, 7, 10, 14, 21, 28, 35, and 42 DPI. Blood, nasal, and throat swabs were collected on all exam dates. Hematology analysis was completed on a ProCyte DX (IDEXX Laboratories, Westbrook, ME, USA) and the following parameters were evaluated: red blood cells (RBC), hemoglobin ( $\mathrm{Hb}$ ), hematocrit (HCT), mean corpuscular volume (MCV), mean corpuscular hemoglobin $(\mathrm{MCH})$, mean corpuscular hemoglobin concentration (MCHC), red cell distribution weight (RDW), platelets, mean platelet volume (MPV), white blood cells (WBC), neutrophil count (abs and \%), lymphocyte count (abs and \%), monocyte count (abs and \%), eosinophil count (abs

244 and \%), and basophil count (abs and \%). Serum chemistries were completed on a VetScan VS2

245 Chemistry Analyzer (Abaxis, Union City, CA) and the following parameters were evaluated: glucose, 
blood urea nitrogen $(\mathrm{BUN})$, creatinine, calcium, albumin, total protein, alanine aminotransferase (ALT), aspartate aminotransferase (AST), alkaline phosphatase (ALP), total bilirubin, globulin, sodium, potassium, chloride, and total carbon dioxide. Ventro-dorsal and right/left lateral radiographs were taken on clinical exam days prior to any other procedures. Radiographs were evaluated and scored for the presence of pulmonary infiltrates by two board-certified clinical veterinarians according to a standard scoring system ${ }^{22}$. Briefly, each lung lobe (upper left, middle left, lower left, upper right, middle right, lower right) was scored individually based on the following criteria: $0=$ normal examination; $1=$ mild interstitial pulmonary infiltrates; 2 = moderate interstitial pulmonary infiltrates, perhaps with partial cardiac border effacement and small areas of pulmonary consolidation (alveolar patterns and air bronchograms); and 3 = pulmonary consolidation as the primary lung pathology, seen as a progression from grade 2 lung pathology. Day 0 radiographs are taken prior to inoculation, and thus serve as a baseline for each animal. All subsequent radiographs are compared to the Day 0 radiographs, evaluated for changes from baseline and scored based on the criteria noted above. At study completion, thoracic radiograph findings are reported as a single cumulative radiograph score for each animal on each exam day; scores may range from 0 to 18. Necropsy was performed on 42 DPI or when euthanasia criteria was reached.

\section{Generation of vaccine ChAdOxl NiV}

ChAdOx1 NiV was produced as previously described ${ }^{20}$. Briefly, the G gene from Nipah virus (Bangladesh outbreak 2008-2010, Genbank accession number: JN808864.1) was codon optimized for humans, synthesized by GeneArt (Thermo Fisher Scientific), and cloned into a transgene expression plasmid comprising a modified human cytomegalovirus immediate early promoter (CMV promoter) with tetracycline operator (TetO) sites and the polyadenylation signal from bovine growth hormone (BGH). This expression cassette was inserted into the E1 locus of the genomic clone of ChAdOx1 using sitespecific recombination $^{38}$. The virus was rescued and propagated in T-REx-293 cells (Invitrogen). Purification was by $\mathrm{CsCl}$ gradient ultracentrifugation, virus titers were determined by hexon immunostaining assay and viral particles were calculated based on spectrophotometry ${ }^{39,40}$.

\section{Cells and virus}

NiV (strain Bangladesh/200401066) was kindly provided by the Special Pathogens Branch of the Centers for Disease Control and Prevention, Atlanta, Georgia, United States. This isolate was obtained from a throat swab collected from patient \#3001 (10-year old male) in Bangladesh on January 22, 2004. The patient developed altered mental status, cough, and breathing difficulties on January 21 . The patient was admitted to Goalando Hospital, Bangladesh, on January 22. None of the patient's contacts developed NiV infection; the patient is presumed to have been infected via direct spillover from the bat reservoir (Dr. Steve Luby, personal communication) ${ }^{41}$. All virus propagation was performed in VeroE6 cells cultured in 
Dulbecco's modified Eagle's medium (DMEM, Sigma) supplemented with 2\% fetal bovine serum (Gibco), $1 \mathrm{mM} \mathrm{L-glutamine} \mathrm{(Gibco),} 50 \mathrm{U} / \mathrm{ml}$ penicillin (Gibco), and $50 \mu \mathrm{g} / \mathrm{ml}$ streptomycin (Gibco) (2\% DMEM). VeroE6 cells were maintained in DMEM supplemented with $10 \%$ fetal calf serum, $1 \mathrm{mM} \mathrm{L}$ glutamine, $50 \mathrm{U} / \mathrm{ml}$ penicillin and $50 \mu \mathrm{g} / \mathrm{ml}$ streptomycin.

285 Virus titrations were performed by end-point titration in VeroE6 cells, which were inoculated with tenfold serial dilutions of virus swab media or tissue homogenates. After $1 \mathrm{hr}$ incubation at $37^{\circ} \mathrm{C}$ and $5 \% \mathrm{CO}_{2}$, tissue homogenate dilutions were removed, washed twice with PBS and replaced with $100 \mu$ 2\% DMEM. Cytopathic effect was scored at 5 DPI and the $\mathrm{TCID}_{50}$ was calculated from a minimum of 4 replicates by the Spearman-Karber method ${ }^{42}$.

\section{Virus neutralization assay}

Heat-inactivated sera $\left(30 \mathrm{~min}, 56{ }^{\circ} \mathrm{C}\right)$ was serially diluted $(2 \mathrm{x})$ in $2 \%$ DMEM. Hereafter, $100 \mathrm{TCID}_{50}$ of $\mathrm{NiV}$ was added. After $1 \mathrm{hr}$ of incubation at $37{ }^{\circ} \mathrm{C}$ and $5 \% \mathrm{CO}_{2}$, serum:virus mixture was added to VeroE6 cells and incubated at $37^{\circ} \mathrm{C}$ and $5 \% \mathrm{CO}_{2}$. At $5 \mathrm{DPI}$, cytopathic effect was scored. The virus neutralization titer was expressed as the reciprocal value of the highest dilution of the serum which still inhibited virus replication.

297 Nipah proteins were produced as previously described ${ }^{20}$. Briefly, NiV-G Malaysia (residues E144 - T602, gene accession number NC_002728) was cloned into the pHLSEC mammalian expression vector ${ }^{43}$ and NiV-F Malaysia (residues G26 - D482, gene accession number AY816748.1) was cloned into the pHLSEC vector containing a C-terminal GCNt trimerization motif ${ }^{44}$. The constructs were transiently expressed in human embryonic kidney (HEK) $293 \mathrm{~T}$ cells. Supernatant was diafiltrated using the AKTA FLUX system (GE Healthcare) against either PBS, pH 7.4 (NiV-G) or buffer containing $10 \mathrm{mM}$ Tris and $150 \mathrm{mM} \mathrm{NaCl}, \mathrm{pH} 8.0$ (NiV-F). The proteins were further purified by Ni-NTA immobilized metalaffinity chromatography using His-Trap HP columns (GE Healthcare) followed by size exclusion chromatography. NiV-G was purified using a Superdex 200 10/300 Increase GL column (GE healthcare) equilibrated in PBS pH 7.4 and NiV-F was purified using a Superose 6 Increase 10/300 GL column (GE healthcare) equilibrated in $10 \mathrm{mM}$ Tris and $150 \mathrm{mM} \mathrm{NaCl} \mathrm{pH} \mathrm{8.0.}$

309 Maxisorp plates (Nunc) were coated overnight at $4{ }^{\circ} \mathrm{C}$ with $5 \mu \mathrm{g}$ of G, N (Native Antigen Company) or F

310 protein per plate in Carb/Bicarb binding buffer (4.41 $\mathrm{g} \mathrm{KHCO}_{3}$ and $0.75 \mathrm{~g} \mathrm{Na}_{2} \mathrm{CO}_{3}$ in $1 \mathrm{~L}$ distilled water).

311 After blocking with 5\% milk in PBS with 0.01\% tween (PBST), serum in 5\% milk in PBST was

312 incubated at RT for $1 \mathrm{hr}$. Antibodies were detected using affinity-purified antibody peroxidase-labeled 
goat-anti-monkey IgG (Seracare) in 5\% milk in PBST and TMB 2-component peroxidase substrate (Seracare) and read at $450 \mathrm{~nm}$. All wells were washed 3x with PBST in between steps.

\section{ELISpot assay and ICS analysis}

PBMCs were isolated from ethylene diamine tetraaceticacid (EDTA) whole blood using LeucosepTM tubes (Greiner Bio-one International $\mathrm{GmbH}$ ) and Histopaque ${ }^{\circledR}-1077$ density gradient cell separation medium (Sigma-Aldrich) according to the manufacturers' instructions. The ImmunoSpot ${ }^{\circledR}$ Human IFN- $\gamma$ Single-Color Enzymatic ELISpot Assay Kit was utilized according to the manufacturer's protocol (Cellular Technology Limited). PBMCs were plated at a concentration of 300,000 cells per well and were stimulated with six contiguous peptide pools spanning the length of the $\mathrm{G}$ protein sequence at a concentration of $2 \mu \mathrm{g} / \mathrm{mL}$ per peptide. One peptide (sequence AFNTVIALLGSIVII) was excluded due to false positive results. Analysis was performed using the CTL ImmunoSpot ${ }^{\circledR}$ Analyzer and ImmunoSpot ${ }^{\circledR}$ Software (Cellular Technology Limited). Spot forming units (SFU) per 1.0x10 $10^{6}$ BMCs were summed across the 6 peptide pools for each animal.

\section{Histology and in situ hybridization}

Harvested tissues were fixed for a minimum of 7 days in $10 \%$ neutral-buffered formalin and subsequently embedded in paraffin. Hematoxylin and eosin (H\&E) staining and in situ hybridization (ISH) were performed on tissue sections and cell blocks. Detection of NiV viral RNA was performed using the RNAscope FFPE assay (Advanced Cell Diagnostics Inc., Newark, USA) as previously described ${ }^{45}$ and in accordance with the manufacturer's instructions. Briefly, tissue sections were deparaffinized and pretreated with heat and protease before hybridization with target-specific probes for $\mathrm{NiV}$ or $\mathrm{HeV}$. Ubiquitin $\mathrm{C}$ and the bacterial gene, dapB, were used as positive and negative controls, respectively. Whole-tissue sections for selected cases were stained for NiV and HeV viral RNA, UBC and dapB by the RNAscope VS FFPE assay (RNAscopeVS, Newark, USA) using the Ventana Discovery XT slide autostaining system (Ventana Medical Systems Inc., Tucson, USA). A board-certified veterinary anatomic pathologist blinded to the study groups evaluated all tissue slides. Pathology score was determined by scoring 6 lung lobes for each animal for the following characteristics: lymphoid cuffing; pneumonia, bronchointerstitial, with fibrin, edema and syncytial cells; and perivascular and alveolar edema and fibrin. Scoring was as follows: $0=$ no lesions; $1=1-10 \% ; 2=11-25 \% ; 3=26-50 \% ; 4=51-$ $75 \% ; 5=76-100 \%$. All scores per animal were added to allow a maximum score of 90 .

\section{Statistical analysis}

Kruskal-Wallis test for multiple comparisons was used to test for statistical significance. P-values $<0.05$ were significant. 


\section{Supplementary Materials}

347 Fig. S1. Presence of infectious virus in non-respiratory or -brain tissue of African green monkeys

348 inoculated with Nipah virus.

349 Table S1. Clinical signs in AGMs inoculated with NiV-B.

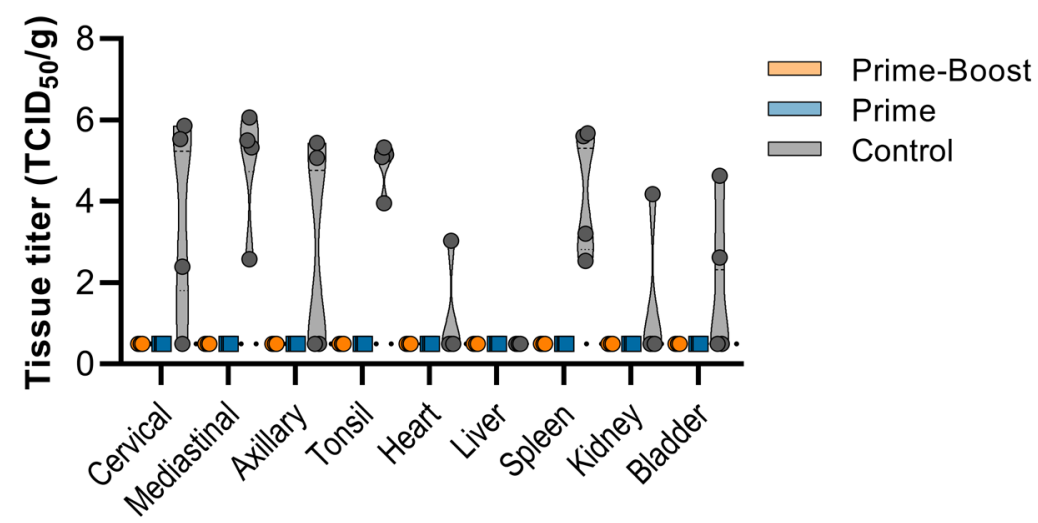

Figure S1. Presence of infectious virus in non-respiratory or brain tissue of African green monkeys inoculated with Nipah virus. Violin plot of infectious virus detected in respiratory tract and brain tissue. Orange circles, prime-boost vaccine; blue squares, prime-only vaccine; grey triangles, controls. No statistical tests were performed since samples were obtained on different days. 


\begin{tabular}{|c|c|c|c|c|c|c|c|c|}
\hline Treatment & Animal & 1 & 2 & 3 & 4 & 5 & 6 & 7 \\
\hline \multirow{4}{*}{$\begin{array}{l}\text { Prime-Boost } \\
\text { ChAdOx1 } \\
\text { NiV }\end{array}$} & 1 & $\mathrm{RA}, 3$ & RA, 3 & $\mathrm{RA}, 3$ & $\mathrm{RA}, 3$ & $\mathrm{RA}, 3$ & $\mathrm{RA}, 5$ & $\mathrm{RA}, 5$ \\
\hline & 2 & RA, 5 & RA, 5 & $\mathrm{RA}, 5$ & $\mathrm{RA}, 5$ & $\mathrm{RA}, 3$ & $\mathrm{RA}, 5$ & $\mathrm{RA}, 3$ \\
\hline & 3 & $\mathrm{RA}, 5$ & $\mathrm{RA}, 5$ & $\mathrm{RA}, 5$ & $\mathrm{RA}, 5$ & $\mathrm{RA}, 3$ & $\mathrm{RA}, 5$ & $\mathrm{RA}, 5$ \\
\hline & 4 & $\mathrm{RA}, 5$ & RA, 5 & $\mathrm{RA}, 5$ & $\mathrm{RA}, 3$ & $\mathrm{RA}, 3$ & $\mathrm{RA}, 3$ & $\mathrm{RA}, 3$ \\
\hline \multirow{4}{*}{$\begin{array}{l}\text { Prime } \\
\text { ChAdOx1 } \\
\text { NiV }\end{array}$} & 5 & $\mathrm{RA}, 3$ & RA, 8 & $\mathrm{RA}, 8$ & $\mathrm{RA}, 3$ & $\mathrm{RA}, 3$ & $\mathrm{RA}, 5$ & $\mathrm{RA}, 5$ \\
\hline & 6 & $\mathrm{RA}, 3$ & $\mathrm{RA}, 10$ & RA, 10 & RA, 5 & $\mathrm{RA}, 5$ & $\mathrm{RA}, 5$ & $\mathrm{RA}, 3$ \\
\hline & 7 & $\mathrm{RA}, 3$ & RA, 5 & $\mathrm{RA}, 3$ & $\mathrm{RA}, 5$ & RA, 3 & $\mathrm{RA}, 5$ & $\mathrm{RA}, 5$ \\
\hline & 8 & $\mathrm{RA}, 5$ & RA, 5 & $\mathrm{RA}, 5$ & $\mathrm{RA}, 3$ & $\mathrm{RA}, 5$ & $\mathrm{RA}, 5$ & $\mathrm{RA}, 5$ \\
\hline \multirow[t]{4}{*}{$\begin{array}{l}\text { Prime-Boost } \\
\text { ChAdOx1 } \\
\text { GFP }\end{array}$} & 9 & $\begin{array}{l}\mathrm{RA}, \mathrm{RF}, \\
3\end{array}$ & $\begin{array}{l}\text { RA, RF, } \\
5\end{array}$ & $\begin{array}{l}\mathrm{RA}, \mathrm{RF}, \\
3\end{array}$ & $\begin{array}{l}\text { RA, RF, } \\
8\end{array}$ & $\begin{array}{l}\text { RA, RF, } \\
5\end{array}$ & $\begin{array}{l}\text { RA, HP, RF, } \\
\text { ND, IR } 25\end{array}$ & $\begin{array}{l}\text { RA, HP, RF, } 40 \\
\text { Fever } 40.3^{\circ} \mathrm{C}, \mathrm{ND}, \mathrm{IR}, \mathrm{OM} \text {, } \\
\mathrm{CY}\end{array}$ \\
\hline & 10 & RA, 5 & RA, 5 &,- 0 & $\mathrm{RA}, \mathrm{IR}, 8$ & IR,5 & $\begin{array}{l}\text { RA, RF, IR, } \\
\mathrm{NS}, \\
25\end{array}$ & HP, RF, RA, IR, NS, 35 \\
\hline & 11 & $\mathrm{RA}, 5$ & $\mathrm{RA}, 5$ & $\mathrm{RA}, 5$ & $\begin{array}{l}\text { RA, } \\
\text { IR, } 10\end{array}$ & $\begin{array}{l}\text { HP, RA, } \\
\text { IR,NS ,40 }\end{array}$ & $\mathrm{n} / \mathrm{a}$ & \\
\hline & 12 & RA,5 & RA,5 & $\begin{array}{l}\text { RA, } \\
\text { IR, } 10\end{array}$ & $\begin{array}{l}\text { RA, RF, } \\
\text { IR, } 15\end{array}$ & $\begin{array}{l}\text { RA, RF, } \\
\text { IR, } 15\end{array}$ & $\begin{array}{l}\text { RA, RF, HP, } \\
\text { ND, IR, OM } \\
\text { CY, } 40\end{array}$ & $\mathrm{n} / \mathrm{a}$ \\
\hline
\end{tabular}

Table S1. Clinical signs in AGMs inoculated with NiV-B. RA = reduced appetite; RF = ruffled fur; $\mathrm{HP}=$ hunched posture; ND= nasal discharge; $\mathrm{IR}=$ increased respirations, $\mathrm{OM}=$ open mouth breathing, $\mathrm{CY}=$ cyanotic, $\mathrm{NS}=$ neurological symptoms 


\section{References and Notes:}

1. Chua, K. B. et al. Nipah virus: a recently emergent deadly paramyxovirus. Science $\mathbf{2 8 8}, 1432-$ 1435 (2000).

2. McKee, C. D. et al. The Ecology of Nipah Virus in Bangladesh: A Nexus of Land-Use Change and Opportunistic Feeding Behavior in Bats. Viruses 13, (2021).

3. ProMED. PRO/AH/EDR> Nipah virus - Bangladesh (02) 20200203.6950171. http://www.promedmail.org/post/6950171.

4. Arunkumar, G. et al. Outbreak Investigation of Nipah Virus Disease in Kerala, India, 2018. J Infect Dis 219, 1867-1878 (2019).

5. Chua, K. B. et al. Isolation of Nipah virus from Malaysian Island flying-foxes. Microbes Infect 4, 145-151 (2002).

6. Rahman, S. A. et al. Characterization of Nipah virus from naturally infected Pteropus vampyrus bats, Malaysia. Emerg Infect Dis 16, 1990-1993 (2010).

7. Reynes, J.-M. et al. Nipah virus in Lyle's flying foxes, Cambodia. Emerg Infect Dis 11, 10421047 (2005).

8. Luby, S. P. et al. Foodborne transmission of Nipah virus, Bangladesh. Emerg Infect Dis 12, $1888-1894$ (2006).

9. Rahman, M. A. et al. Date palm sap linked to Nipah virus outbreak in Bangladesh, 2008. Vector Borne Zoonotic Dis 12, 65-72 (2012).

10. Islam, M. S. et al. Nipah Virus Transmission from Bats to Humans Associated with Drinking Traditional Liquor Made from Date Palm Sap, Bangladesh, 2011-2014. Emerg Infect Dis 22, 664-670 (2016). 
11. Halpin, K. et al. Pteropid bats are confirmed as the reservoir hosts of henipaviruses: a comprehensive experimental study of virus transmission. Am J Trop Med Hyg 85, 946-951 (2011).

12. Gurley, E. S. et al. Person-to-person transmission of Nipah virus in a Bangladeshi community. Emerg Infect Dis 13, 1031-1037 (2007).

13. Nikolay, B. et al. Transmission of Nipah Virus - 14 Years of Investigations in Bangladesh. N Engl J Med 380, 1804-1814 (2019).

14. Weatherman, S., Feldmann, H. \& de Wit, E. Transmission of henipaviruses. Curr Opin Virol 28, 7-11 (2018).

15. Amaya, M. \& Broder, C. C. Vaccines to Emerging Viruses: Nipah and Hendra. Annu Rev Virol 7, 447-473 (2020).

16. Broder, C. C., Weir, D. L. \& Reid, P. A. Hendra virus and Nipah virus animal vaccines. Vaccine 34, 3525-3534 (2016).

17. Geisbert, T. W. et al. A single dose investigational subunit vaccine for human use against Nipah virus and Hendra virus. npj Vaccines 6, 23 (2021).

18. Pritchard, E. et al. Impact of vaccination on new SARS-CoV-2 infections in the United Kingdom. Nat Med (2021) doi:10.1038/s41591-021-01410-w.

19. Public Health England press office. Vaccines highly effective against hospitalisation from Delta variant. https://www.gov.uk/government/news/vaccines-highly-effective-againsthospitalisation-from-delta-variant.

20. van Doremalen, N. et al. A single-dose ChAdOx1-vectored vaccine provides complete protection against Nipah Bangladesh and Malaysia in Syrian golden hamsters. PLoS Negl Trop Dis 13, e0007462 (2019). 
21. Geisbert, T. W. et al. Development of an Acute and Highly Pathogenic Nonhuman

Primate Model of Nipah Virus Infection. PLoS ONE 5, e10690 (2010).

22. Brining, D. L. et al. Thoracic radiography as a refinement methodology for the study of H1N1 influenza in cynomologus macaques (Macaca fascicularis). Comp Med 60, 389-395 (2010).

23. Bossart, K. N. et al. A Hendra Virus G Glycoprotein Subunit Vaccine Protects African Green Monkeys from Nipah Virus Challenge. Science Translational Medicine 4, 146ra107146ra107 (2012).

24. Lo, M. K. et al. Single-dose replication-defective VSV-based Nipah virus vaccines provide protection from lethal challenge in Syrian hamsters. Antiviral Research 101, 26-29 (2014).

25. Prescott, J. et al. Single-dose live-attenuated vesicular stomatitis virus-based vaccine protects African green monkeys from Nipah virus disease. Vaccine 33, 2823-2829 (2015).

26. Price, A et al. Nipah Virus Assays and Animal Models for Vaccine Development. https://media.tghn.org/medialibrary/2021/02/Nipah_Virus_Assays_and_Animal_Models_for_ Vaccine_Development_final.pdf.

27. Snoy, P. J. Establishing Efficacy of Human Products Using Animals: The US Food and Drug Administration’s “Animal Rule”. Vet Pathol 47, 774-778 (2010).

28. U.S. Food \& Drug. Animal Rule Approvals. https://www.fda.gov/drugs/nda-and-blaapprovals/animal-rule-approvals (2021).

29. Voysey, M. et al. Safety and efficacy of the ChAdOx1 nCoV-19 vaccine (AZD1222) against SARS-CoV-2: an interim analysis of four randomised controlled trials in Brazil, South 
Africa, and the UK. The Lancet S0140673620326611 (2020) doi:10.1016/S0140-

$6736(20) 32661-1$.

30. Mire, C. E. et al. A Recombinant Hendra Virus G Glycoprotein Subunit Vaccine Protects

Nonhuman Primates against Hendra Virus Challenge. Journal of Virology 88, 4624-4631

(2014).

31. Yoneda, M. et al. Recombinant Measles Virus Vaccine Expressing the Nipah Virus

Glycoprotein Protects against Lethal Nipah Virus Challenge. PLoS ONE 8, e58414 (2013).

32. Mire, C. E. et al. Use of Single-Injection Recombinant Vesicular Stomatitis Virus

Vaccine to Protect Nonhuman Primates Against Lethal Nipah Virus Disease. Emerg. Infect.

Dis. 25, 1144-1152 (2019).

33. van Doremalen, N. et al. A single dose of ChAdOx1 MERS provides protective immunity in rhesus macaques. Sci. $A d v$. 6, eaba8399 (2020).

34. van Doremalen, N. et al. ChAdOx1 nCoV-19 vaccine prevents SARS-CoV-2 pneumonia in rhesus macaques. Nature (2020) doi:10.1038/s41586-020-2608-y.

35. Feldmann, F., Shupert, W. L., Haddock, E., Twardoski, B. \& Feldmann, H. Gamma Irradiation as an Effective Method for Inactivation of Emerging Viral Pathogens. Am J Trop Med Hyg 100, 1275-1277 (2019).

36. Haddock, E., Feldmann, F. \& Feldmann, H. Effective Chemical Inactivation of Ebola Virus. Emerg Infect Dis 22, 1292-1294 (2016).

37. Munster, V. J. et al. Respiratory disease in rhesus macaques inoculated with SARS-CoV2. Nature 585, 268-272 (2020). 
38. Dicks, M. D. J. et al. A Novel Chimpanzee Adenovirus Vector with Low Human

Seroprevalence: Improved Systems for Vector Derivation and Comparative Immunogenicity.

PLoS ONE 7, e40385 (2012).

39. Bewig, B. \& Schmidt, W. E. Accelerated Titering of Adenoviruses. BioTechniques 28, $870-873$ (2000).

40. Maizel, J. V., White, D. O. \& Scharff, M. D. The polypeptides of adenovirus. Virology

36, 115-125 (1968).

41. de Wit, E. et al. Foodborne Transmission of Nipah Virus in Syrian Hamsters. PLoS

Pathog 10, e1004001 (2014).

42. Kärber, G. Beitrag zur kollektiven Behandlung pharmakologischer Reihenversuche.

Archivf. experiment. Pathol. u. Pharmakol 162, 480-483 (1931).

43. Aricescu, A. R., Lu, W. \& Jones, E. Y. A time- and cost-efficient system for high-level protein production in mammalian cells. Acta Crystallogr D Biol Crystallogr 62, 1243-1250 (2006).

44. Chan, Y.-P. et al. Biochemical, Conformational, and Immunogenic Analysis of Soluble Trimeric Forms of Henipavirus Fusion Glycoproteins. Journal of Virology 86, 11457-11471 (2012).

45. Wang, F. et al. RNAscope. The Journal of Molecular Diagnostics 14, 22-29 (2012).

Acknowledgments: We thank the animal caretakers, Rachel LaCasse, Danielle Adney, Thomas A. Bowden, Anita Mora, Robert Fischer, Myndi Holbrook, Ilona Rissanen, Emmie de Wit, and Trenton Bushmaker for their assistance during the study. Funding: This work was supported by the Division of Intramural Research of the National Institute of Allergy and Infectious Diseases (NIAID), National Institutes of Health (NIH) and CEPI (award reference: 276871). Author contributions: N.v.D., T.L., S.C.G. and V.J.M. designed the study; N.v.D., V.A.A., R.F., J.E.S., 
E.H., A.O., J.L., P.W.H., K.C., G.S, and V.J.M. acquired, analyzed and interpreted the data; N.v.D. and V.J.M. wrote the manuscript. All authors have approved the submitted version.

Competing interests: S.C.G. is a board member of Vaccitech and named as an inventor on a patent covering the use of $\mathrm{ChAdOx} 1$-vector-based vaccines and a patent application covering a SARS-CoV-2 (nCoV-19) vaccine (UK patent application no. 2003670.3). T.L. is named as an inventor on a patent application covering a SARS-CoV-2 (nCoV-19) vaccine (UK patent application no. 2003670.3). The University of Oxford and Vaccitech, having joint rights in the vaccine, entered into a partnership with AstraZeneca in April 2020 for further development, large-scale manufacture and global supply of the vaccine. Equitable access to the vaccine is a key component of the partnership. Neither Oxford University nor Vaccitech will receive any royalties during the pandemic period or from any sales of the vaccine in developing countries. The other authors declare no competing interests. Data and materials availability: If data are in an archive, include the accession number or a placeholder for it. Here also include any materials that must be obtained through an MTA. Acknowledgments follow the references and notes but are not numbered. 